Reprod. Nutr. Dévelop., 1987, 27 (1 B), 317-318.

\title{
Influence de la lipomobilisation \\ sur la sécrétion des triglycérides par le foie, chez le mouton
}

\author{
A. MAZUR, M. AL-KOTOBE, Y. RAYSSIGUIER
}

Laboratoire des Maladies Métaboliques, I.N.R.A. Theix, 63122 Ceyrat, France.

Summary. The relationship between hepatic triglyceride secretion and plasma free fatty acids was determined in sheep using Triton WR 1339 to block lipoprotein lipase activity.

La relation existant entre la lipomobilisation et la sécrétion de triglycérides par le foie est intéressante à étudier chez le Ruminant puisque les acides gras libres plasmatiques sont les précurseurs des triglycérides hépatiques et qu'une sécrétion insuffisante de lipoprotéines pourrait être à l'origine de stéatose hépatique lors d'une forte lipomobilisation. II est possible de mesurer cette sécrétion par l'utilisation d'un détergent non ionique, le Triton WR 1339, qui bloque spécifiquement l'action de la lipoprotéine lipase (Otway et Robinson, 1967). Compte tenu de la faible contribution des lipides alimentaires, l'élévation des triglycérides plasmatiques après injection de Triton reflète essentiellement la sécrétion par le foie. Nous avons étudié celle-ci chez le mouton, soumis à des conditions physiologiques et nutritionnelles variées, de façon à obtenir des intensités de lipomobilisation très différentes.

Matériel et méthodes. Les animaux de race Limousine $\times$ Romanov recevant en bergerie une alimentation de type foin et concentré étaient utilisés dans les conditions suivantes (nombre d'animaux) : mâles castrés nourris (3), brebis non gestantes nourries (10), à jeun depuis 1 jour (4), à jeun depuis 3 jours (9) ; brebis gestantes nourries (3) ou à jeun depuis 1 jour (4). Le Triton 1339 (Tyloxapol Sigma) dilué dans du sérum physiologique (15\% poids/volume) était injecté par voie intraveineuse à la dose de $0,2 \mathrm{~g} / \mathrm{kg}$ de poids corporel. Des prélèvements sanguins étaient effectués immédiatement avant l'injection puis 6 heures après de façon à déterminer le taux d'acides gras libres avant traitement et l'évolution des triglycérides plasmatiques. Les acides gras libres et les triglycérides étaient dosés par voie enzymatique. Le calcul de la sécrétion de triglycérides était effectué selon la formule de Schultz et Esdale (1971) :

$$
\begin{aligned}
& \text { Sécrétion des triglycérides }=\frac{C_{2}-C_{1}}{t_{2}-t_{1}} \times K \\
& (\mathrm{mg} / \mathrm{kg} / \mathrm{min})
\end{aligned}
$$

où $\mathrm{C}_{1}$ et $\mathrm{C}_{2}=$ concentrations des triglycérides exprimées en $\mathrm{mg}$ de triglycérides par $\mathrm{ml}$ de plasma avant et $6 \mathrm{~h}$ après l'injection de Triton; $t_{1}$ et $t_{2}=$ temps des prélèvements en minutes $(0$ et $360 \mathrm{~min}) ; \mathrm{K}=$ constante équivalente à $40 \mathrm{ml}$ de plasma par $\mathrm{kg}$ de poids corporel.

Résultats et discussion. L'injection de Triton entraîne un aspect lactescent du plasma lié à une forte triglycéridémie. La relation existant entre la sécrétion de triglycérides $(Y, \mathrm{mg} / \mathrm{min} / \mathrm{kg}$ p.c.) et le taux d'acides gras libres plasmatiques $(X$, $\mathrm{mM})$ peut être représentée par une courbe qui a pour équation :

$$
Y=0,1758 e^{0,2382 \log (x)} \quad r=0,6844
$$


Alors que la mise à jeun diminue la lipogenèse hépatique et la sécrétion de triglycérides par le foie chez le Monogastrique (Otway et Robinson, 1967), elle augmente la réestérification des acides gras dans le foie et la sécrétion de triglycérides chez le Ruminant (Mamo, Snoswell et Topping, 1983). Cependant cette sécrétion n'est pas proportionnelle au taux circulant d'acides gras libres et reste relativement limitée lors de forte lipomobilisation. Ce résultat est à mettre en rapport avec la fréquence de l'infiltration lipidique du foie chez le Ruminant lors de lipolyse intense (mise à jeun prolongée, fin de gestation chez la brebis, début de lactation chez la vache haute productrice) et confirmerait la capacité limitée du foie à émettre des lipoprotéines légères riches en triglycérides (Rayssiguier et al., 1986).

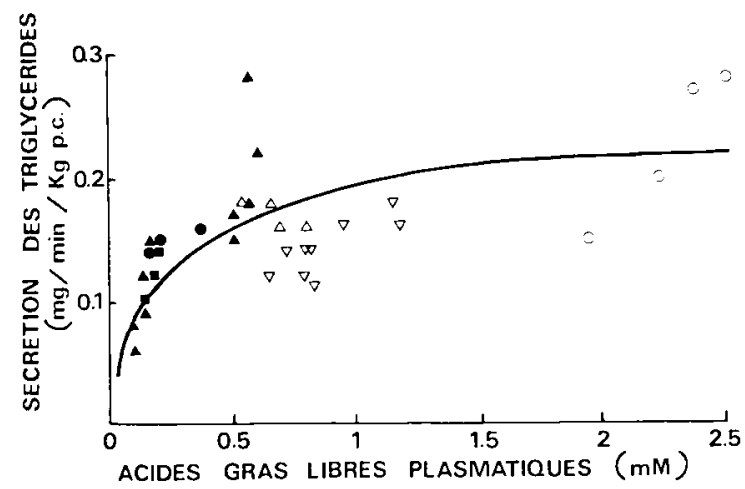

FIG. 1. - Relation entre le taux des acides gras libres plasmatiques et la sécrétion des triglycérides chez les ovins. Brebis vides : nourries $(\boldsymbol{\Delta})$, à jeun depuis 1 jour $(\Delta)$, à jeun depuis 3 jours $(\nabla)$; brebis dans le dernier mois de gestation: nourries $(\bullet)$, à jeun depuis 1 jour $(0)$; mâles castrés nourris ( $\mathbf{E})$.

Mamo J. C. L., Snoswell A. M., Topping D. L., 1983. Biochim. Biophys. Acta, 753, 272-275.

Otway S., Robinson D. S., 1967. J. Physiol., 190, 321-332.

Rayssiguier Y., Mazur A., Reid I. M., Roberts C. J., Gueux E., 1986. Reprod. Nutr. Dévelop., 26, $361-362$.

Schultz L. H., Esdale W. J., 1971. J. Dairy Sci, 54, 1173-1179. 